\title{
Performance Analysis of Designing a Hybrid Optical Amplifier (HOA) for 32 DWDM Channels in L-band by using EDFA and Raman Amplifier
}

\author{
Aied K. Mohammed, PhD \\ Department of Electrical Engineering, \\ University of Technology
}

\author{
Noor Yahya Jawad \\ Department of Electrical Engineering, \\ University of Technology
}

\begin{abstract}
In this paper, the hybrid amplifier has been design and simulated using Optisystem Software version 13. This design of HOA using two types of optical fiber amplifiers such, Raman amplifier and Erbium Doped Fiber Amplifier (EDFA) to amplifying 32 channels in L-band (1560-1600 nm). The pumping power and pumping wavelength for the two types of amplifiers are the same and equal to $300 \mathrm{mw}$ and $1490 \mathrm{~nm}$ respectively, the EDFA and Raman amplifier are using backward pumping configuration. The overall gain and noise of this design is $38.52 \pm 1.28 \mathrm{~dB}$ and $4.5 \pm 0.7 \mathrm{~dB}$ respectively without using any flatting technique. But by using a new flatting technique based on Gaussian filter between the Raman and EDFA the gain ripple enhanced to $38.46 \pm 0.55 \mathrm{~dB}$ with noise $4.5 \pm 0.7 \mathrm{~dB}$ over the L-band in the optical communication system spectrum.
\end{abstract}

\section{Keywords}

EDFA, Raman Amplifier, Hybrid optical amplifier, DWDM, L-band.

\section{INTRODUCTION}

In long-haul point-to-point optical fiber communication the signal traveling inside the fiber suffers from various losses like fiber attenuation losses, fiber tap losses, fiber splice losses, etc., due to these losses it is difficult to detect the original signal at the receiver side. So in order to transmit signal over a long distance in a fiber it is important to compensate all losses in the fiber [1]. They can be done either by using optoelectronic repeaters or optical amplifiers. Optoelectronic repeaters have previously been used to compensate the power losses. In it, the optical signal first converted into an electric signal, then amplified in electric domain and finally converted back to optical signals. The use of such repeaters in optical communication systems have made the systems more complex and expensive for wavelength division multiplexed (WDM) in light wave systems [2, 3].

Optical amplifiers, as their name implies, operate solely in the optical domain with no inter-conversion of photons to electrons [4]. The signal is optically amplified in strength by several orders of magnitude without being limited by any electronic bandwidth. The shift from regenerators to amplifiers thus permits a dramatic increase in capacity of the transmission system. Optical amplifiers play an important role in increasing the capacity of optical communication systems using such amplifiers [5]. Optical amplifiers have been essential elements for high capacity, long-lifespan and multiple connection of optical for communication network applications [6]. There are several types of optical amplifier such Semiconductor optical amplifier, EDFA, Raman amplifier, and parametric optical amplifier.

\section{EDFA}

An important class of lumped optical amplifiers makes use of rare-earth elements as a gain medium by doping the fiber core during the manufacturing process. Although doped-fiber amplifiers were studied as early as 1964 , their use became practical only 25 years later, after their fabrication techniques were perfected. In such amplifiers, properties such as the operating wavelength and the gain bandwidth are determined by the dopants while silica plays the role of a host medium [7].

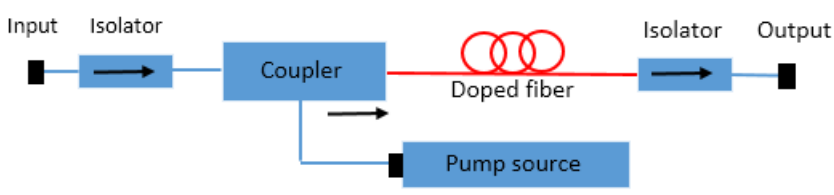

Fig 1: general component of rare earth amplifier

The rare-earth ions that used in doping of optical fiber are Erbium (Er), Ytterbium (Yb), Thulium (Th), Praseodymium $(\mathrm{Pr})$, and Neodymium $(\mathrm{Nd})$. Each one of these used at certain wavelength band.

The Erbium-Doped Fiber Amplifier was the first optical amplifier widely used in optical communications systems, it has become a key component in many optical networks because it provides efficient, low-noise amplification of light in the optical fiber low-loss telecommunications window near $1550 \mathrm{~nm}[8,9]$.

\section{RAMAN AMPLIFIER}

Nonlinear effects within optical fiber may also be employed to provide optical amplification. Such amplification can be achieved by using stimulated Raman scattering (SRS) [4]. So, Raman amplification has been one of the optical amplifier technologies that had a slow start, but then experienced a wide deployment with increasing performance needs of optical networks [10]. They can be operated over a range of telecommunications windows, from below $1300 \mathrm{~nm}$ to beyond $1650 \mathrm{~nm}$, often with broader spectra than those of erbium doped fiber amplifier.

The gain medium can be transmission fiber or dispersion compensating fiber (DCF). DCF-based Raman amplifiers simultaneously boost the propagating signals and compensate for accumulated chromatic dispersion [11]. Raman amplifiers utilize pumps to impart a transfer of energy from the pumps to the transmission signals through the Raman Effect mechanisms [10]. 


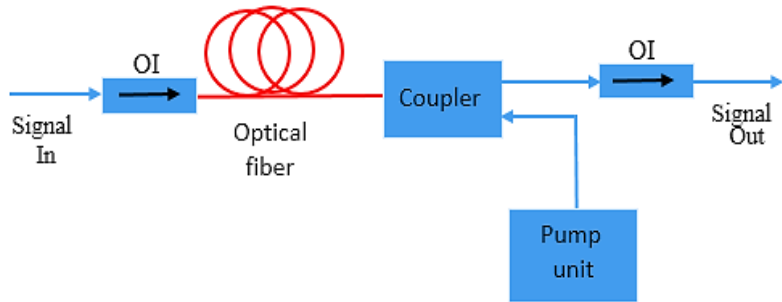

Fig 3: Raman amplifiers with a counter-propagating [46]

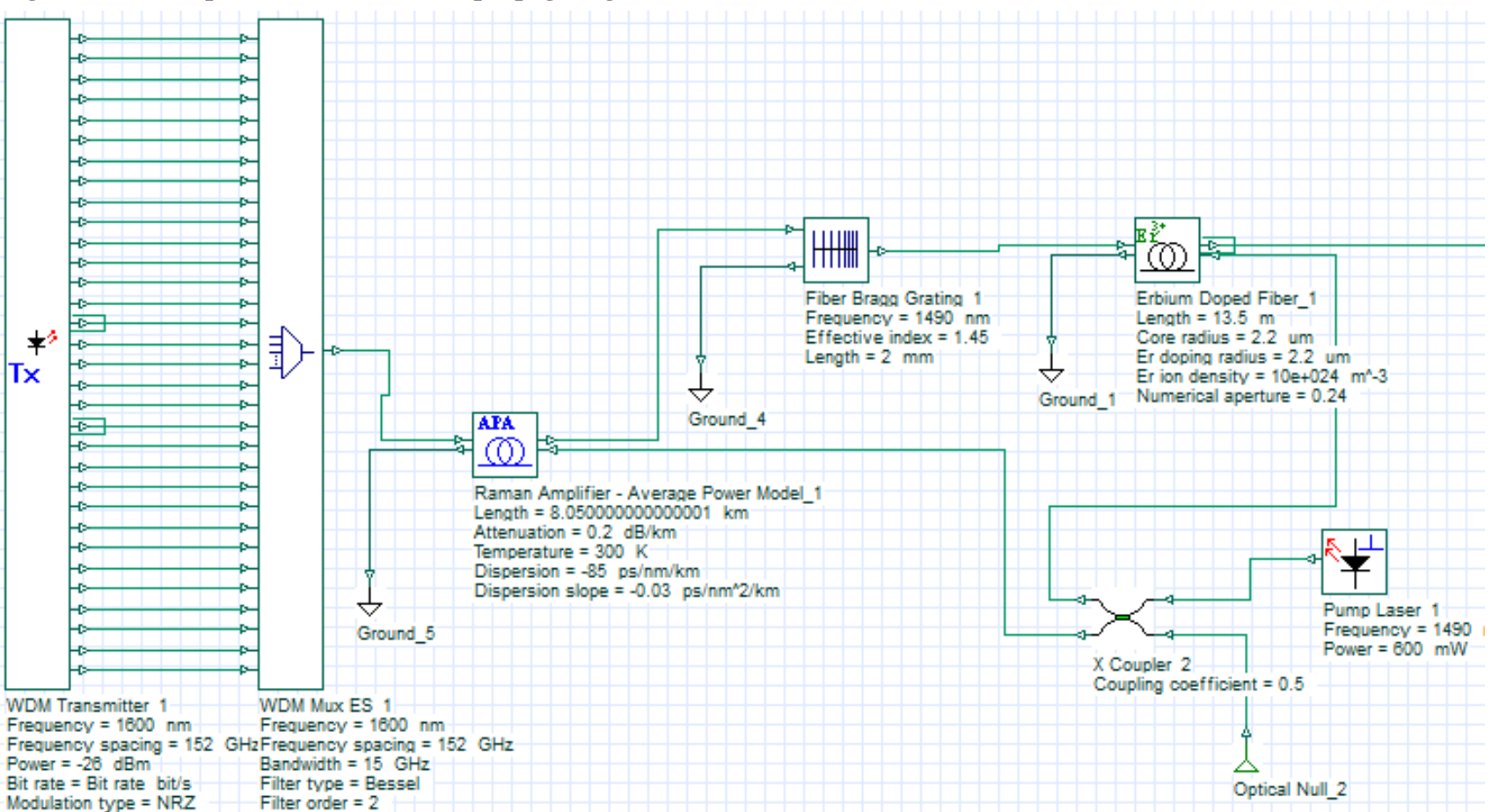

Fig 3: Hybrid amplifier for L- band

\section{DESIGN AND SIMULATION}

The two types of amplifiers (Raman amplifier and EDFA) is used to amplifying 32 channels in L-band region (1560-1600 $\mathrm{nm})$.

The following tables show the specifications of the Raman amplifier and EDFA

\section{Table 1: EDFA specifications}

\begin{tabular}{|l|l|}
\hline Parameter & Value \\
\hline Length & $13.5 \mathrm{~m}$ \\
\hline Core radius & $2.2 \mathrm{um}$ \\
\hline Doping radius & $2.2 \mathrm{um}$ \\
\hline Numerical appercher(NA) & 0.24 \\
\hline Concentration & $10 e^{+24} \mathrm{~m}^{-3}$ \\
\hline Pumping power & $300 \mathrm{mw}$ \\
\hline Pumping wavelength & $1490 \mathrm{~nm}$ \\
\hline
\end{tabular}

The pump signal optical wavelengths in Raman fiber amplifiers are typically $500 \mathrm{~cm}-1$ higher in frequency than the signal to be amplified, and the continuous-wave Raman gains exceeding $20 \mathrm{~dB}$ in silica fiber. The basic physical idea behind Raman scattering is an interaction of an incident light wave with intrinsic vibrations of molecules. When a light wave is propagating in the medium, it scatters, creating a phonon through excitation of the molecule vibrations [12]. 


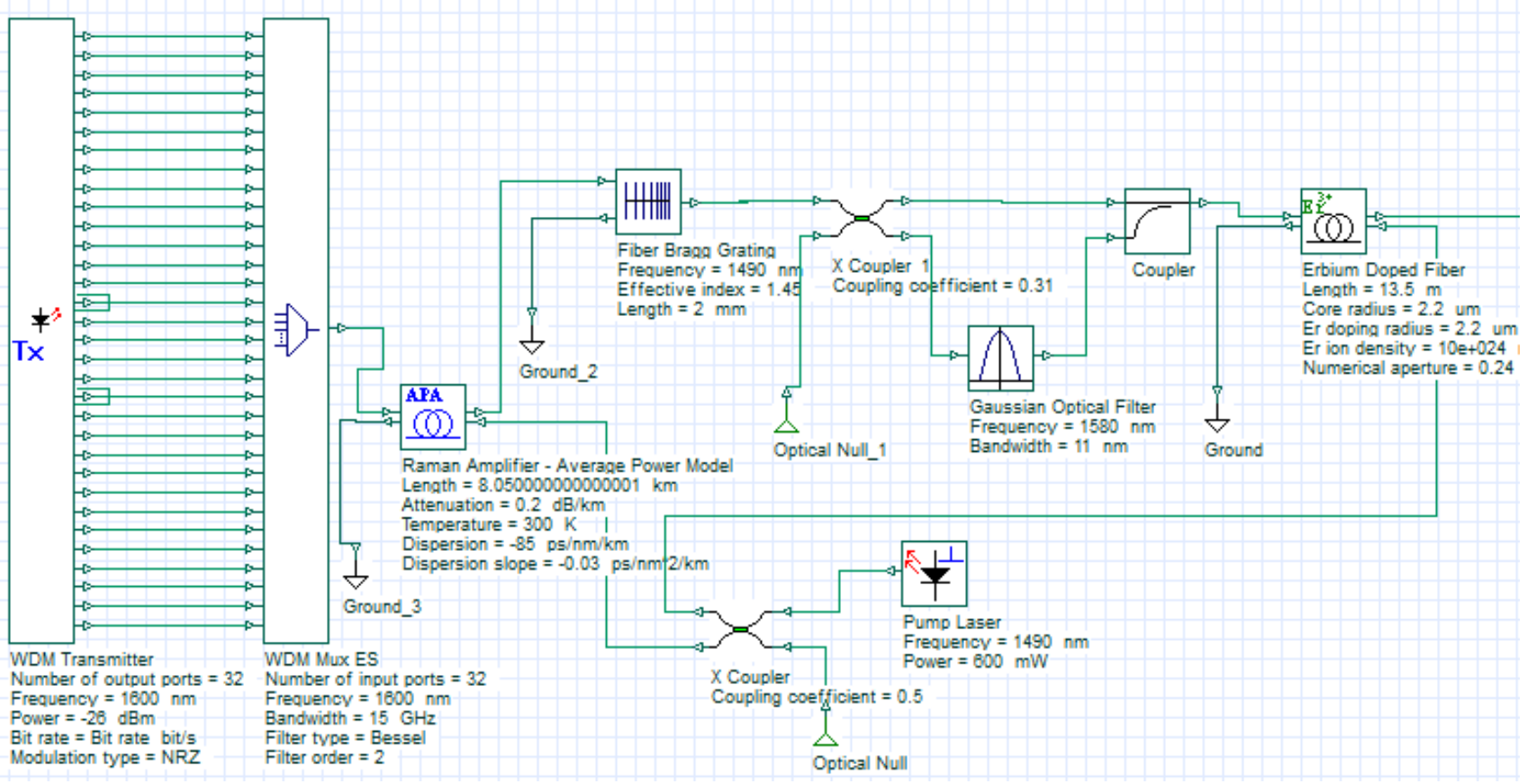

Fig 4: Hybrid amplifier for L-band with flatting technique in between the two amplifiers.

The purpose of this design is to get flat gain over wide range of frequencies. The Gaussian band pass filter (BPF) used for flatting a certain region of L-band. This process done by divided the signal into two branches by using X-coupler with 0.31 coupling coefficient. The second branch connect to the BPF which has center wavelength of $1580 \mathrm{~nm}$ with bandwidth $11 \mathrm{~nm}$, after the BPF, the coupler used to combine these branches as shown in Fig.(4).

The hybrid amplifier design that shown in Fig. (4) is used in optical communication system to amplifying 32 channels for nearly $1120 \mathrm{~km}$. in this optical communication system there are 8 spans, each span consists of $140 \mathrm{~km}$ single mode fiber (SMF) with an attenuation value of $0.2 \mathrm{~dB} / \mathrm{km}$, a dispersion coefficient value of $16 \mathrm{ps} / \mathrm{nm} / \mathrm{km}$ and dispersion slope coefficient of $0.075 \mathrm{ps} / \mathrm{nm}^{2} / \mathrm{km}$ followed by a DCF fiber with a length of $19.5 \mathrm{~km}$, its attenuation constant is $0.5 \mathrm{~dB} / \mathrm{km}$, the dispersion coefficient value is $-85 \mathrm{ps} / \mathrm{nm} / \mathrm{km}$ and the dispersion slope coefficient is $-0.3 \mathrm{ps} / \mathrm{nm}^{2} . \mathrm{km}$. The effective area of the DCF is $22 \mu \mathrm{m}^{2}$.

\section{SIMULATION RESULTS AND DISCASSION}

The proposed design which was given in Fig. (3) has been simulated and the gain variation of EDFA, Raman amplifier, and overall gain as shown in Fig. (5).

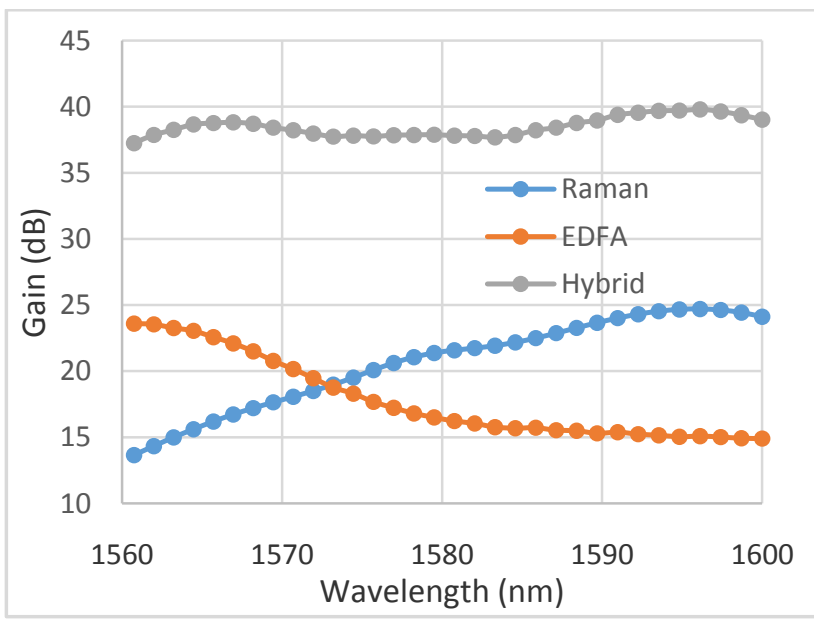

Fig 5: Gain variation of $L$-band hybrid amplifier

The pumping power and wavelength is the same for the EDFA and Raman amplifier, the pumping wavelength $=1490 \mathrm{~nm}$. It was tried to reduce the Raman amplifier length in order to reduce amplifier noise. The gain ripple for this design is $2.55 \mathrm{~dB}$.

To improve flatting gain the design shown in Fig. (4) That used flatting technique is simulated, Fig. (6) Show the gain comparison between the first and second design. 


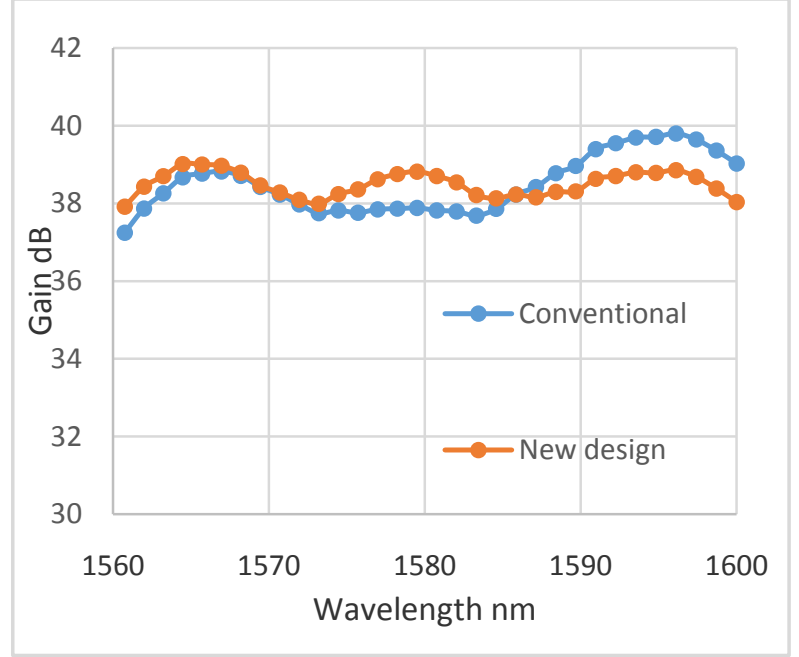

Fig 6: Gain variation of hybrid amplifier at L-band for different design configurations

This flatting technique reduce the gain ripple to $1.09 \mathrm{~dB}$.

Figure below show the noise figure at different design configurations, the noise figure is low in spite of the Raman amplifier at first stage because the length of Raman amplifier is small which is equal to $8 \mathrm{~km}$ that produce low value of noise.

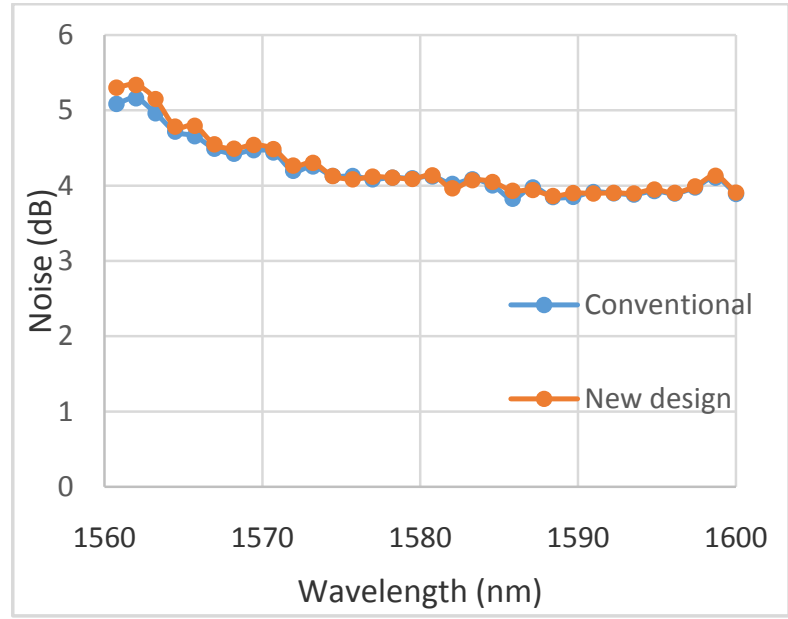

Fig 7: Noise figure variation of hybrid amplifier at L-band for different design configurations

As shown there is no effect on noise performance in two designs which indicate this flatting technique improve the gain flatting and in the same time it doesn't increase the noise of amplifier system.

Fig. (8) Shows the signals before and after $1120 \mathrm{~km}$.

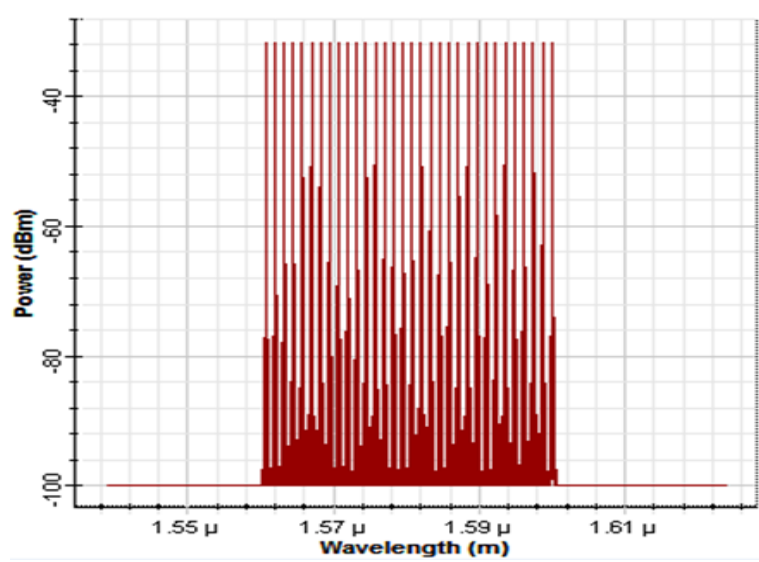

(a)

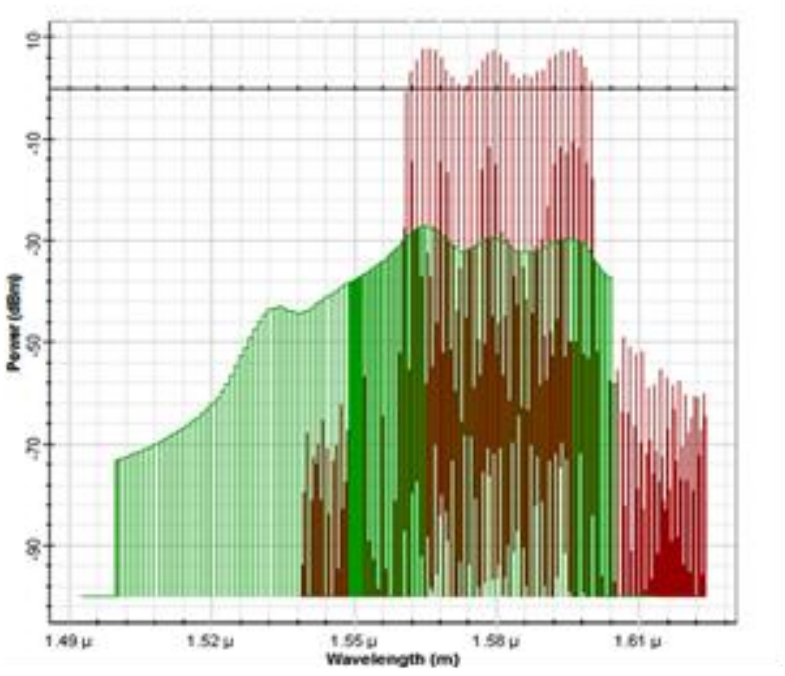

(b)

Fig 8: Signal power (a) Transmitted signal channel (b) Received signals after $1120 \mathrm{~km}$

The noise spectrum (green color in (b)) appear after $1120 \mathrm{~km}$ due noise from the optical amplifier Raman and EDFA, these noise add at each amplification stage making the OSNR decrease. The difference between signals power after $1120 \mathrm{~km}$ is about $8 \mathrm{dBm}$ because we have eight stages of hybrid amplifier and each stage provide about $1 \mathrm{~dB}$ gain ripple.

The bit error rate and quality factor for the first channel and the eighteenth channel after $1120 \mathrm{~km}$ are $5.98 * 10^{-17}, 8.24$, $1.26 * 10^{-18}$ and 8.71 respectively. Fig. (9) Show the eye diagram for the two channels. 


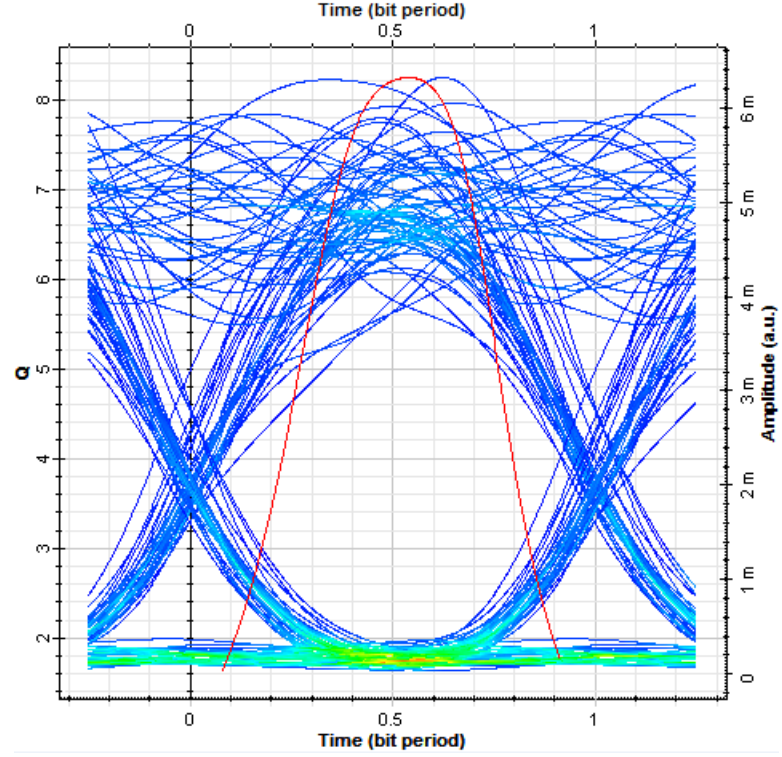

(a)

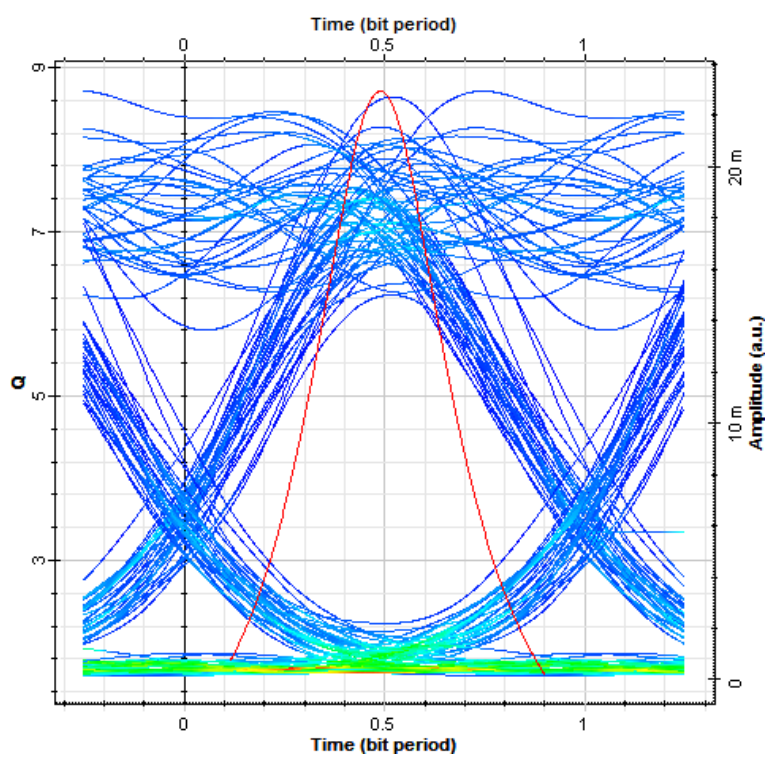

(b)

Fig 9: Eye diagram after 1120km (a) for the first channel (b) for the eighteenth channel

\section{CONCLUSIONS}

a new design of the hybrid amplifier (Raman follow by EDFA) are suggested for L-band using the same pumping power and pumping wavelength $300 \mathrm{mw}$ and $1490 \mathrm{~nm}$ respectively for two types of optical fiber amplifier (Raman and EDFA), a small length of Raman amplifier was used to reduce the noise. This design provide high gain $38.52 \pm 1.28$
$\mathrm{dB}$, and by using mid-stage flatting technique with the Gaussian filter, the gain flatness enhanced to $38.46 \pm 0.55 \mathrm{~dB}$ with noise figure $4.5 \pm 0.7 \mathrm{~dB}$. This hybrid optical amplifier design used in the optical communication system for $1120 \mathrm{~km}$ and provide good Bit Error Rate after this distance. For future it can be increase the number of channels, and using another rare earth optical amplifier with Raman amplifier.

\section{REFERENCES}

[1]. P. Shukla, K. P. Kaur, "Performance Analysis of EDFA for different Pumping Configurations at High Data Rate", International Journal of Engineering and Advanced Technology (IJEAT), Volume 2, Issue 5, June 2013.

[2]. A. C. Çokrak, A. Altuncu, "Gain and noise figure performance of Erbium Doped Fiber Amplifier (EDFA)", Istanbul University - Journal of Electrical \& Electronic Engineering, Volume 4, number 2, pp. 11111122, 2004.

[3]. P. kaur, S. Kaur, G. Singh, "Speed Enhancement of network using EDFA optical amplifier", International Journal of Electrical, Electronic and Mechanical Controls, Volume 3, Issue 1, January 2014.

[4]. C. F. Lam, "Passive Optical Networks: Principles and Practice" Academic Press, 2007.

[5]. Lekshmi.S.R, Sindhu.N, "Spectral Response of FWM in EDFA for Long-haul Optical Communication", International Journal of Innovative Research in Computer and Communication Engineering Vol. 2, Issue 10, pp. 6091-6095, October 2014.

[6]. M.A.Othman , M.M. Ismail , M.H.Misran, M.A.M.Said and H.A.Sulaiman, "Erbium Doped Fiber Amplifier (EDFA) for C-Band Optical Communication System", International Journal of Engineering \& Technology IJET-IJENS, Vol 12, No. 4, pp. 48-50, 2012.

[7]. G. P. Agrawal, "Fiber-Optic Communication Systems", John Wiley \& Sons, Fourth Edition, 2002.

[8]. M. J. F. Digonnet, "Rare Earth Doped Fiber Lasers and Amplifiers" Marcel Dekker, 2001.

[9]. J. Zyskind, and A. Srivastava, "Optically Amplified WDM Networks", Elsevier, 2011.

[10]. M. N. Islam, "Raman Amplifiers for Telecommunications 1 Physical Principles", Springer, 2004.

[11]. P. Urquhart, "Advances in Optical Amplifiers", InTech, 2011.

[12]. V. Ter-Mikirtychev, "Fundamentals of Fiber Lasers and Fiber Amplifiers", Springer, 2014 